\title{
Expression changes of CX3CL1 and CX3CR1 proteins in the hippocampal CA1 field of the gerbil following transient global cerebral ischemia
}

\author{
JI HYEON AHN ${ }^{1 *}$, DAE WON KIM ${ }^{2 *}$, JOON HA PARK ${ }^{1}$, TAE-KYEONG LEE ${ }^{3}$, \\ HYANG-AH LEE ${ }^{4}$, MOO-HO WON ${ }^{3}$ and CHOONG-HYUN LEE ${ }^{5}$
}

\begin{abstract}
${ }^{1}$ Department of Biomedical Science, Research Institute of Bioscience and Biotechnology, Hallym University, Chuncheon, Gangwon 24252; ${ }^{2}$ Department of Biochemistry and Molecular Biology, and Research Institute of

Oral Sciences, College of Dentistry, Gangnung-Wonju National University, Gangneung, Gangwon 25457; Departments of ${ }^{3}$ Neurobiology and ${ }^{4}$ Obstetrics and Gynecology, School of Medicine, Kangwon National University, Chuncheon, Gangwon 24341; ${ }^{5}$ Department of Pharmacy, College of Pharmacy, Dankook University,

Cheonan, Chungcheongnam 31116, Republic of Korea
\end{abstract}

Received March 19, 2019; Accepted July 8, 2019

DOI: $10.3892 /$ ijmm.2019.4273

\begin{abstract}
Chemokine C-X3-C motif ligand 1 (CX3CL1) and its sole receptor, CX3CR1, are known to be involved in neuronal damage/death following brain ischemia. In the present study, time-dependent expression changes of CX3CL1 and CX3CR1 proteins were investigated in the hippocampal CA1 field following $5 \mathrm{~min}$ of transient global cerebral ischemia (tgCI) in gerbils. To induce tgCI in gerbils, bilateral common carotid arteries were occluded for $5 \mathrm{~min}$ using aneurysm clips. Expression changes of CX3CL1 and $\mathrm{CX} 3 \mathrm{CR} 1$ proteins were assessed at 1,2 and 5 days after $\operatorname{tgCI}$ using western blotting and immunohistochemistry. CX3CL1 immunoreactivity was strong in the CA1 pyramidal cells of animals in the sham operation group. Weak CX3CL1 immunoreactivity was detected at $6 \mathrm{~h}$ after $\operatorname{tgCI}$, recovered at 1 day after $\operatorname{tgCI}$ and disappeared from 5 days after tgCI. CX3CR1 immunoreactivity was very weak in CA1 pyramidal cells of the sham animals. CX3CR1 immunoreactivity in CA1 pyramidal cells was significantly increased at 1 days after tgCI and gradually decreased thereafter. On the other hand, CX3CR1 immunoreactivity was significantly increased in microglia
\end{abstract}

Correspondence to: Professor Moo-Ho Won, Department of Neurobiology, School of Medicine, Kangwon National University, 1 Gangwondaehak, Chuncheon, Gangwon 24341, Republic of Korea E-mail:mhwon@kangwon.ac.kr

Professor Choong-Hyun Lee, Department of Pharmacy, College of Pharmacy, Dankook University, 119 Dandae-ro, Cheonan, Chungcheongnam 31116, Republic of Korea

E-mail: anaphy@dankook.ac.kr

*Contributed equally

Key words: CX3CL1, CX3CR1, delayed neuronal death, microglial activation, transient global cerebral ischemia from 5 days after tgCI. These results showed that CX3CL1 and CX3CR1 protein expression levels in pyramidal cells and microglia in the hippocampal CA1 field following tgCI were changed, indicating that tgCI-induced expression changes of CX3CL1 and CX3CR1 proteins might be closely associated with tgCI-induced delayed neuronal death and microglial activation.

\section{Introduction}

It is well known that brain injury and ischemic insults cause neuronal damage/death by increasing intracellular calcium concentration, glutamate-mediated excitotoxicity and oxidative stress following ischemic events (1-4). In addition, overactivation of neuroinflammatory responses including microglial activation and increases of proinflammatory cytokines can lead to brain injury including neuronal death/loss (5-8).

Chemokines, as regulators of brain inflammation, are known to be detrimental factors following brain ischemic insults because their overexpression can increase ischemia-induced brain injuries and recruitment of inflammatory cells (9-11). Among various chemokines, chemokine C-X3-C motif ligand 1 (CX3CL1, also called as fractalkine) as one of $\mathrm{CX} 3 \mathrm{C}$ chemokines is constitutively expressed in the central nervous system. It is especially localized in neurons (12). CX3C chemokine receptor 1 (CX3CR1), a G-protein-coupled receptor, is the sole receptor for CX3CL1. Certain previous studies have demonstrated that it is expressed in microglia (12-14). It has widely been accepted that the CX3CL1/CX3CR1 signaling pathway plays important roles in regulating cellular interactions between neurons and microglia in the brain $(12,15)$. In addition, CX3CL1/CX3CR1 pathway has been thought to be associated with the activation and recruitment of microglia (15-17). CX3CL1 is known to suppress microglial activation and CX3CR1 plays an important role in modulating normal microglial activity by inhibiting microglial activity (18-20). 
Previous studies have reported ischemia-induced changes of CX3CL1 and/or CX3CR1 expression in rodent brains following focal cerebral ischemia $(17,21-23)$. However, the expression and roles of CX3CL1 and CX3CR1 in the brain following transient global cerebral ischemia (tgCI) in rodents have not been fully elucidated yet. It is known that $\operatorname{tgCI}$ in the brain is caused by impaired blood flow, leading to deprivation of oxygen and glucose and resulting in selective neuronal death/damage in vulnerable brain regions including the hippocampus $(24,25)$. In the hippocampus, pyramidal cells in the CA1 field are known to be vulnerable to tgCI. Death of CA1 pyramidal cells induced by tgCI is called 'delayed neuronal death (DND)' because CA1 pyramidal cells will die a few days after $\operatorname{tgCI}$ for $5 \mathrm{~min}$ (24). Therefore, the objective of the present study was to investigate time-dependent changes of CX3CL1 and CX3CR1 expression in neuronal and/or glial cells of the gerbil hippocampal CA1 field following $5 \mathrm{~min}$ of $\operatorname{tgCI}$.

\section{Materials and methods}

Experimental animals. Male Mongolian gerbils (Meriones unguiculatus), aged 6 months (body weight $\sim 68-73 \mathrm{~g}$; $\mathrm{n}=84$ ), were obtained from the Experimental Animal Center, Kangwon National University, (Chuncheon, Republic of Korea). The animals were housed in a conventional state under adequate temperature $\left(23^{\circ} \mathrm{C}\right)$ and humidity $(60 \%)$ control, with a 12-h light/12-h dark cycle and were provided with free access to food and water. Experimental procedures for this study were approved by the Institutional Animal Care and Use Committee at Kangwon National University (approval number: KW-180124-1). In this study, numbers of animals used and the suffering caused by the procedures used in this experiment were minimized.

$\operatorname{tg}$ CI induction. According to the method of the authors previous studies $(7,26,27)$, the induction of $\operatorname{tg} C$ I was performed. In brief, the gerbils were anesthetized with a mixture of isoflurane $(2.5 \%)$ in oxygen $(30 \%)$ and nitrous oxide (70\%), with a modification of methods of previous studies (28-30) and level of anesthesia was confirmed by pedal reflex (firm toe pinch). Bilateral common carotid arteries were occluded for $5 \mathrm{~min}$ using aneurysm clips. The complete interruption of blood flow was confirmed by observing the central artery in the retina using an ophthalmoscope (Heine Optotechnik). Their rectal temperature $\left(37 \pm 0.5^{\circ} \mathrm{C}\right)$ was kept using a thermometric blanket during and after $\operatorname{tgCI}$. Sham operated gerbils were subjected to the same procedure without bilateral common carotid artery occlusion.

Western blotting. CX3CL1 and CX3CR1 protein levels in the CA1 field were analyzed at designated times (6 h, 1, 2, 5 and 10 days after $\operatorname{tg} \mathrm{CI}$ ) using western blot method. According to the authors' published method (7), in short, 7 animals at each point in time were anaesthetized with sodium pentobarbital (60 mg/kg, i.p.) and their brains were removed. Their brains were serially and transversely cut into $400-\mu \mathrm{m}$ thickness using a vibratome (Leica Camera AG; Leica Microsystems, Inc.). CA1 fields were dissected with a surgical blade and homogenized in $50 \mathrm{mM}$ phosphate-buffered saline (PBS;
$\mathrm{pH}$ 7.4) containing $0.1 \mathrm{mM}$ ethylene glycol bis (2-aminoethyl ether)-N,N,N0,N0 tetraacetic acid (pH 8.0), 0.2\% Nonidet $\mathrm{P}-40,10 \mathrm{mM}$ ethylendiamine tetraacetic acid ( $\mathrm{pH} 8.0), 15 \mathrm{mM}$ sodium pyrophosphate, $100 \mathrm{mM} \beta$-glycerophosphate, $50 \mathrm{mM}$ $\mathrm{NaF}, 150 \mathrm{mM} \mathrm{NaCl}, 2 \mathrm{mM}$ sodium orthovanadate, $1 \mathrm{mM}$ phenylmethylsulfonyl fluoride and $1 \mathrm{mM}$ dithiothreitol (DTT). The homogenized tissues were centrifugated at $16,000 \mathrm{x} \mathrm{g}$ for $20 \mathrm{~min}$ at $4^{\circ} \mathrm{C}$ and protein levels of CX3CL1 and CX3CR1 were determined using a Micro BCA protein assay kit (Pierce Chemical, Co.). Aliquot containing total protein $(20 \mathrm{mg})$ was boiled in loading buffer containing $150 \mathrm{mM}$ Tris-HCI (pH 6.8), $6 \%$ SDS, 3 mM DTT, $0.3 \%$ bromophenol blue and $30 \%$ glycerol and loaded onto $10 \%$ polyacrylamide gel. The gel was transferred to nitrocellulose transfer membranes (Pall Corporation) after electrophoresis. The background of the membrane was reduced with 5\% nonfat dry milk in PBS containing $0.1 \%$ Tween-20 for $40 \mathrm{~min}$ at room temperature and the membrane was reacted with rabbit anti-CX3CL1 (cat. no. NBP1-49539; 1:1,000; Novus Biologicals, LLC) or mouse anti-CX3CR1 (cat. no. 824001; 1:1,000; BioLegend Inc.) overnight at $4^{\circ} \mathrm{C}$. Followed by incubation with peroxidase-conjugated goat anti-rabbit or mouse IgG (cat nos. ab6721 and ab205719; 1:5,000; Abcam) for $1 \mathrm{~h}$ at room temperature and visualization with a Pierce ECL western blotting substrate (cat. no. 32106; Thermo Fisher Scientific, Inc.). The loading control was carried out using mouse anti- $\beta$-actin antibody (cat. no. A5316; $1: 5,000$; Sigma-Aldrich; Merck KGaA). Western blot analysis using homogenates at all experimental time-points was performed simultaneously. Results of the western blotting were scanned and the quantification of the bands was densitometrically analyzed using ImageJ 1.46 software (National Institutes of Health). The quantification was represented by relative optical density (ROD). A ratio of the ROD was calibrated as \%: The sham operated gerbil was designated as $100 \%$.

Preparation of histological sections. For cresyl violet (CV) staining, fluoro-jade B (FJB) histofluorescence, immunohistochemical and double immunofluorescence stainings, brain sections containing the hippocampus were prepared from the sham and $\operatorname{tgCI}$ operated gerbils ( $\mathrm{n}=7$ at each point in time) at designated times ( 6 h, 1, 2, 5 and 10 days after tgCI). According to the authors' published method $(7,26,27)$, the gerbils were anaesthetized with sodium pentobarbital and perfused transcardially with $0.1 \mathrm{M}$ PBS followed by $4 \%$ paraformaldehyde. And then, their brains were removed and postfixed with the same fixative for $8 \mathrm{~h}$ at room temperature and cryoprotected by infiltration with $30 \%$ sucrose for $10 \mathrm{~h}$. The tissues were serially sectioned into $30-\mu \mathrm{m}$ frontal sections in a cryostat (Leica Microsystems).

In addition, sham operated tissue was presented only at 5 days after $\operatorname{tgCI}$, because there were no significant differences between the sham control samples at each designated time (data not shown).

$C V$ staining. $\mathrm{CV}$ histochemical staining was performed to investigate cellular distribution and morphology. In brief, according to the authors' published method (31), CV acetate (Sigma-Aldrich; Merck KGaA) was dissolved (1\%) in distilled water (DW) and glacial acetic acid was added to this solution. Sections of each group were stained with CV solution and 
dehydrated with serial ethanol. The sections were examined using an AxioM1 light microscope (Carl Zeiss AG).

FJB histofluorescence staining. FJB [a marker for neurodegeneration (32)] histofluorescence staining was performed to examine $\operatorname{tg} C \mathrm{C}$-induced DND in the CA1 field. As previously described $(7,26,27)$, in brief, the sections were serially stained with a $1 \%$ sodium hydroxide solution, a $0.06 \%$ potassium permanganate solution and a $0.0004 \%$ FJB (cat. no. AG301; Merck $\mathrm{KGaA}$ ) solution at room temperature. The reacted sections were examined using an epifluorescent microscope (Carl Zeiss AG) equipped with a blue excitation light (450-490 $\mathrm{nm})$ and a barrier filter.

Immunohistochemistry. Immunohistochemical staining was carried out for ionized calcium-binding adapter molecule 1 (Iba1; a marker for microglia), CX3CL1 and CX3CR1. According to the authors' previous studies $(7,26,27)$, in brief, the sections were incubated with rabbit anti-Ibal (cat. no. 019-19741; 1:800; Wako Pure Chemical Industries, Ltd.) for microglia, rabbit anti-CX3CL1/Fractalkine (cat. no. NBP1-49539; 1:250; Novus Biologicals, LLC), or mouse anti-CX3CR1 (cat. no. 824001; 1:100; BioLegend Inc.) as primary antibodies overnight at $4^{\circ} \mathrm{C}$. The reacted sections were exposed to biotinylated goat anti-rabbit $\mathrm{IgG}$ or horse anti-mouse IgG (cat. nos. BA-1000 and BA-2000; 1:250; Vector Laboratories) for $1 \mathrm{~h}$ at room temperature and streptavidin peroxidase complex. Finally, the reacted sections were visualized with 3,3'-diaminobenzidine at room temperature.

In order to confirm the specificity of each immunoreaction, each negative control test was done using pre-immune serum (Vector Laboratories) instead of each primary antibody. Each negative control test showed no immunoreactivity in each immunostained tissue. In addition, immunohistochemical staining was performed simultaneously at all experimental time-points.

Double immunofluorescence staining. To examine cell types containing CX3CR1 immunoreactivity, double immunofluorescence staining was performed according to the authors' published protocol (27). In brief, mouse anti-CX3CR1 (cat. no. 824001; 1:50; BioLegend Inc.)/rabbit anti-Iba1 (cat. no. 019-19741; 1:400; Wako Pure Chemical Industries, Ltd.) were used for microglia. The sections were incubated in the mixture of the antisera overnight at $4^{\circ} \mathrm{C}$ and the incubated sections were reacted in a mixture of both goat anti-mouse IgG, Alexa Fluor488 (cat. no. A-11001; 1:500; Invitrogen; Thermo Fisher Scientific, Inc.) and donkey anti-rabbit IgG, Alexa Fluor546 (cat. no. A10040; 1:500; Invitrogen; Thermo Fisher Scientific, Inc.) for $1 \mathrm{~h}$ at room temperature. The immunoreaction was observed under a confocal microscope (LSM510 META NLO; Carl Zeiss AG) in the Korea Basic Science Institute Chuncheon Center.

Data analyses. Data analyses were performed by two or three investigators, who were blind to the experimental conditions. First, the numbers of $\mathrm{CV}$ and FJB positive cells were analyzed according to the authors' published method (27). In brief, 8 sections were selected from each animal with $120-\mu$ m interval according to antero-posterior -1.4 to $-2.2 \mathrm{~mm}$ of the gerbil brain atlas. Images of $\mathrm{CV}$ and FJB positive cells were captured with an AxioM1 light microscope (Carl Zeiss AG) equipped with a digital camera (Axiocam; Carl Zeiss AG) connected to a PC monitor. Cells were obtained in a $250 \times 250 \mu \mathrm{m}$ square and the cell counts were obtained by averaging the total number of $\mathrm{CV}$ and FJB positive cells from each animal per group using an image analyzing system (software: Optimas 6.5; CyberMetrics Corporation).

For quantitative analyses of Iba1, CX3CL1 and CX3CR1 immunoreactivities, 8 sections were selected with $120-\mu \mathrm{m}$ interval in each gerbil. Each immunoreactive image in the CA1 field was captured with an AxioM1 light microscope (Carl Zeiss AG) equipped with a camera (Axiocam; Carl Zeiss AG) connected to a PC monitor. According to the authors' published method $(7,26,27)$, each image was captured in a corresponding area $(250 \times 250 \mu \mathrm{m})$ of the CA1 field at $40 \mathrm{X}$ primary magnification and the image was calibrated into an array of $512 \times 512$ pixels. The densities of Iba1, CX3CL1 and CX3CR1 immunoreactive structures were evaluated on the basis of optical density (OD): The OD was obtained after the transformation of the mean gray level using the formula: $O D=\log (256 /$ mean gray level). After the background density was subtracted, a ratio of the OD was calibrated as \% [relative optical density (ROD)] and analyzed using NIH ImageJ 1.59 software (National Institute of Health). A ratio of the ROD was calibrated as \%, with sham tgCI operated gerbils designated as $100 \%$.

Statistical analysis. The data shown in the present study represent the mean \pm standard error of the mean. The normality test was performed using a Kolmogorov and Smirnov test for testing normal distributions, and Bartlett test for testing identical standard distributions. All data passed normality tests. Differences of the means among the groups were statistically analyzed by analysis of variance with Duncan's post hoc test in order to elucidate ischemia-related differences among experimental groups. $\mathrm{P}<0.05$ was considered to indicate a statistically significant difference.

\section{Results}

tgCI-induced changes in CX3CL1 and CX3CR1 protein levels. CX3CL1 and CX3CR1 protein levels in the CA1 field were significantly changed with time after tgCI (Fig. 1). CX3CL1 and CX3CR1 protein levels were significantly decreased by $\sim 65 \%(\mathrm{P}<0.05)$ and $26 \%(\mathrm{P}<0.05)$ at $6 \mathrm{~h}$ after $\operatorname{tgCI}$ compared with the sham operated group. Additionally, compared with the $6 \mathrm{~h}$ group, CX3CL1 and CX3CR1 protein levels were significantly increased by $\sim 165 \%(\mathrm{P}<0.05)$ and $206 \%(\mathrm{P}<0.05)$, respectively at 1 day after $\operatorname{tgCI}$, and compared with the 1 day group, CX3CL1 and CX3CR1 protein levels were significantly decreased by $\sim 130 \%(\mathrm{P}<0.05)$ and $100 \%(\mathrm{P}<0.05)$, respectively, at 2 days after $\operatorname{tgCI}$. At 5 days after $\operatorname{tgCI}$, CX3CL1 protein level was significantly decreased $(\mathrm{P}<0.05)$ by $\sim 35 \%$; however, CX3CR1 protein level was significantly increased $(\mathrm{P}<0.05)$ by $\sim 145 \%$, compared with those at 2 days after tgCI. At 10 days after $\operatorname{tgCI}, \mathrm{CX} 3 \mathrm{CL} 1$ and CX3CR1 protein levels were not significantly different from those at 5 days post-tgCI.

tgCI-induces DND and microglia activation. tgCI-induced DND was examined in the CA1 field at 5 days after tgCI using 

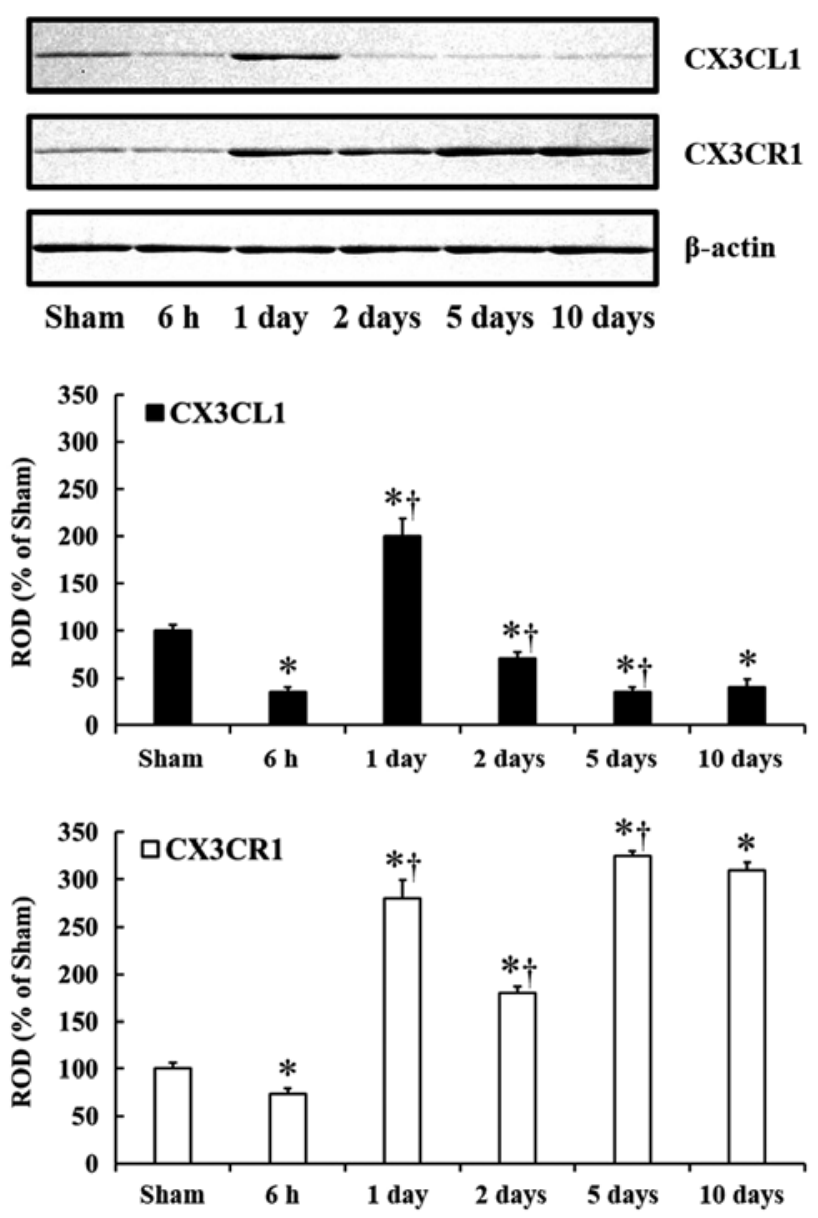

Figure 1. Western blot analyses of CX3CL1 and CX3CR1 in the hippocampal CA1 field of sham operated and ischemia operated gerbils. ROD as \% value of immunoblot band is represented ( $\mathrm{n}=7$ per group). ${ }^{*} \mathrm{P}<0.05$ vs. the sham operated gerbils; ${ }^{\dagger} \mathrm{P}<0.05$ vs. the pre-time-point group. $\mathrm{CX} 3 \mathrm{CL} 1, \mathrm{C}-\mathrm{X} 3-\mathrm{C}$ motif ligand 1 ; CX3CR1, CX3C chemokine receptor 1; ROD, relative optical density.

CV staining and FJB histofluorescence staining (Figs. 2 and 3). $\mathrm{CV}$ staining was shown in cells of the stratum pyramidale, which are called pyramidal neurons, in the CA1-3 field of the sham operated group (Fig. 2A and G). In the ischemia operated group, pyramidal neurons were also well stained with CV until 2 days after tgCI (Fig. 2B, D, H and J); however, numbers of $\mathrm{CV}$ positive pyramidal neurons were significantly decreased $(\mathrm{P}<0.05)$ in the $\mathrm{CA} 1$ field from 5 days after $\operatorname{tgCI}$ (Fig. 2E, F and K-M).

In the sham operated gerbils, no FJB positive cells were found in any layer (Fig. 3A). In addition, FJB positive cells were not observed until 2 days post-tgCI (Fig. 3B-D). However, at 5 and 10 days after tGCI, a number of FJB positive cells were observed in the stratum pyramidale of the CA1 field, not CA2/3 field, and the number of FJB positive cells was significantly increased $(\mathrm{P}<0.05)$ compared with the sham group (Fig. 3E-G).

Significant changes of microglia were found, which are mononuclear phagocytic cells, in the CA1 field after tgCI (Fig.4). Iba1 immunoreactive microglia were observed as a resting form in the sham operated group and they were scattered in all layers of the CA1 field (Fig. 4A). In the ischemia operated group, Iba1 immunoreactivity was gradually increased in a time-dependent manner after $\operatorname{tgCI}$ and Ibal immunoreactivity in all ischemia operated groups was significantly increased $(\mathrm{P}<0.05)$ compared with the sham group. In addition, the microglia were morphologically activated in the ischemia operated group and they were hypertrophied in the cytoplasm and thickened in processes (Fig. 4B-G). Especially, at 5 and 10 days after tgCI, a number of activated Iba1 immunoreactive microglia were aggregated in the stratum pyramidale, where tgCI-induced DND of CA1 pyramidal cells occurred (Fig. 4E and F).

tgCI-induces change in CX3CL1 immunoreactivity. In the sham operated gerbils, CX3CL1 immunoreactivity was mainly observed in CA1 pyramidal cells (Fig. 5A). However, in the ischemia operated gerbils, CX3CL1 immunoreactivity in CA1 pyramidal cells was very weak and significantly decreased $(\mathrm{P}<0.05)$ at $6 \mathrm{~h}$ after $\operatorname{tgCI}$ (Fig. 5B and G). At 1 day after tgCI, CX3CL1 immunoreactivity was very strong in CA1 pyramidal cells, showing that the CX3CL1 immunoreactivity was significantly increased $(\mathrm{P}<0.05,172.2 \%$ of the sham operated gerbils) compared with the sham operated gerbils (Fig. 5C and G). Thereafter, CX3CL1 immunoreactivity in CA1 pyramidal cells began to significantly decrease from 2 days after $\operatorname{tgCI}$ $(\mathrm{P}<0.05,54.7 \%$ of the sham operated gerbils) and CX3CL1 immunoreactivity in the CA1 pyramidal cells was weak at 5 and 10 days after $\operatorname{tgCI}(\mathrm{P}<0.05,21.2 \%$ and $\mathrm{P}<0.05,28.6 \%$ of the sham operated gerbils, respectively), because death of CA1 pyramidal cells occurred after tgCI (Fig. 5D-G).

tgCI-induces change in CX3CRI immunoreactivity. In the sham operated gerbils, weak CX3CR1 immunoreactivity was observed in CA1 pyramidal cells and their processes (Fig. 6A). In the ischemia operated gerbils, CX3CR1 immunoreactivity in the CA1 field at $6 \mathrm{~h}$ after tgCI was significantly decreased $(\mathrm{P}<0.05)$ by $36.5 \%$ compared with the sham operated gerbils (Fig. 6B and G). At 1 day after tgCI, CX3CR1 immunoreactivity in the CA1 field was significantly increased $(\mathrm{P}<0.05)$ by $103 \%$ compared with the sham operated gerbils, showing that the increased CX3CR1 immunoreactivity was shown in CA1 pyramidal cells (Figs. 6C and 5G). At 2 days after tgCI, CX3CR1 immunoreactivity was significantly decreased $(\mathrm{P}<0.05)$ by $25.4 \%$ compared with 1 day after tgCI (Fig. 6D and G). At 5 and 10 days after tgCI, CX3CR1 immunoreactivity in the CA1 field was significantly increased by $203.8 \%(\mathrm{P}<0.05)$ and $189.4 \%$ $(\mathrm{P}<0.05)$, respectively, compared with the sham operated gerbils. At these points in time, strong CX3CR1 immunoreactivity was shown in cells in strata oriens and radiatum (Fig. 6E-G).

tgCI-induces CX3CRI immunoreactivity in microglia. Strong CX3CR1 immunoreactivity was shown in cells in strata oriens and radiatum at 5 and 10 days after tgCI (Fig. $6 \mathrm{E}$ and F). Their cell type was examined using double immunofluorescence staining and found that cells expressing CX3CR1 in strata oriens and radiatum were identified as Iba1 immunoreactive microglia (Fig. 7A-C).

\section{Discussion}

In the present study, strong CX3CL1 immunoreactivity was found mainly in CA1 pyramidal cells in the sham operated gerbils, consistent with a previous study showing that CX3CL1 was constitutively expressed in neurons (12). In addition, 

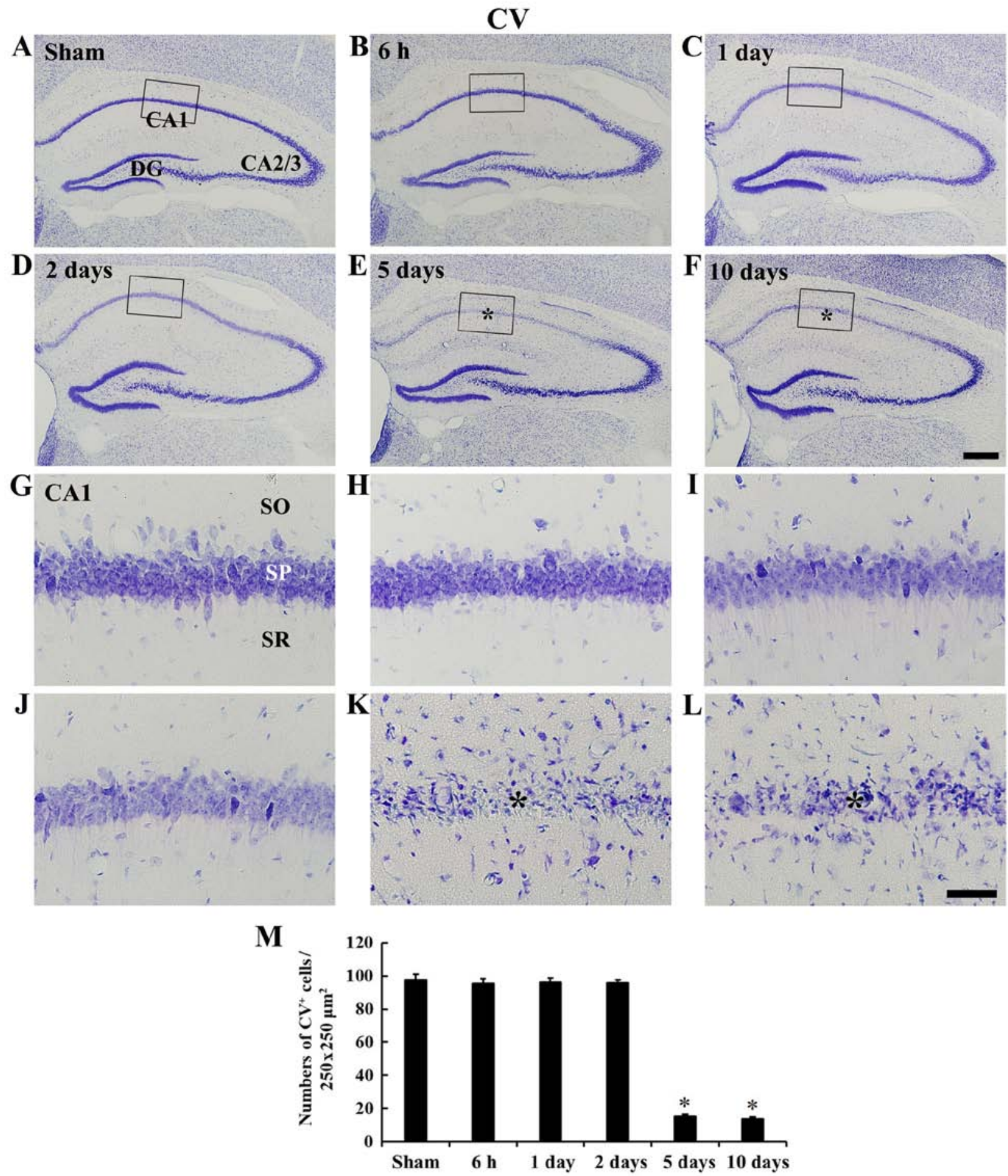

Figure 2. CV staining. Low magnification of the hippocampus of the (A) sham group and ischemia operated groups at (B) $6 \mathrm{~h}$, (C) 1 day, (D) 2 days, (E) 5 days and (F) 10 days after tgCI. High magnification of the CA1 field of the (G) sham group and ischemia operated groups at (H) 6 h, (I) 1 day, (J) 2 days, (K) 5 days, and (L) 10 days after tgCI. CV positive cells are well observed in the SP in the CA1-3 field in the sham operated group. In the ischemia operated group, CV positive cells are significantly decreased only in the SP (asterisks) of the CA1 field at 5 and 10 days after tgCI. Scale bars $=400 \mu \mathrm{m}(\mathrm{A}-\mathrm{F})$ and $50 \mu \mathrm{m}(\mathrm{G}-\mathrm{L})$. (M) Numbers of CV positive cells in the CA1 field ( $\mathrm{n}=7$ at each point in time). $\mathrm{P}<0.05$ vs. the sham operated group. The bars indicate the mean \pm standard error of the mean. CV, cresyl violet; SP, stratum pyramidale; SO, stratum oriens; SR, stratum radiatum; tgCI, transient global cerebral ischemia.

weak CX3CR1 immunoreactivity was found in CA1 pyramidal cells in the sham operated gerbils. Meucci et al (33) reported that CX3CR1 is expressed in hippocampal neurons. They suggested that neuronal CX3CR1 might have mediated neurotrophic effects of fractalkine and that both fractalkine and CX3CR1 might be involved in cellular communications between neurons and glia.
In this study, CX3CL1 and CX3CR1 immunoreactivities were observed in CA1 pyramidal cells that were transiently and reduced at $6 \mathrm{~h}$ after tgCI and then increased at 1 day after $\operatorname{tgCI}$. This result is somewhat consistent with a previous study showing that CX3CL1/CX3CR1 expression is downregulated within 24 after ischemic injury and upregulated at $48 \mathrm{~h}$ to 7 days following focal cerebral ischemia in rats (17). 


\section{FJB}
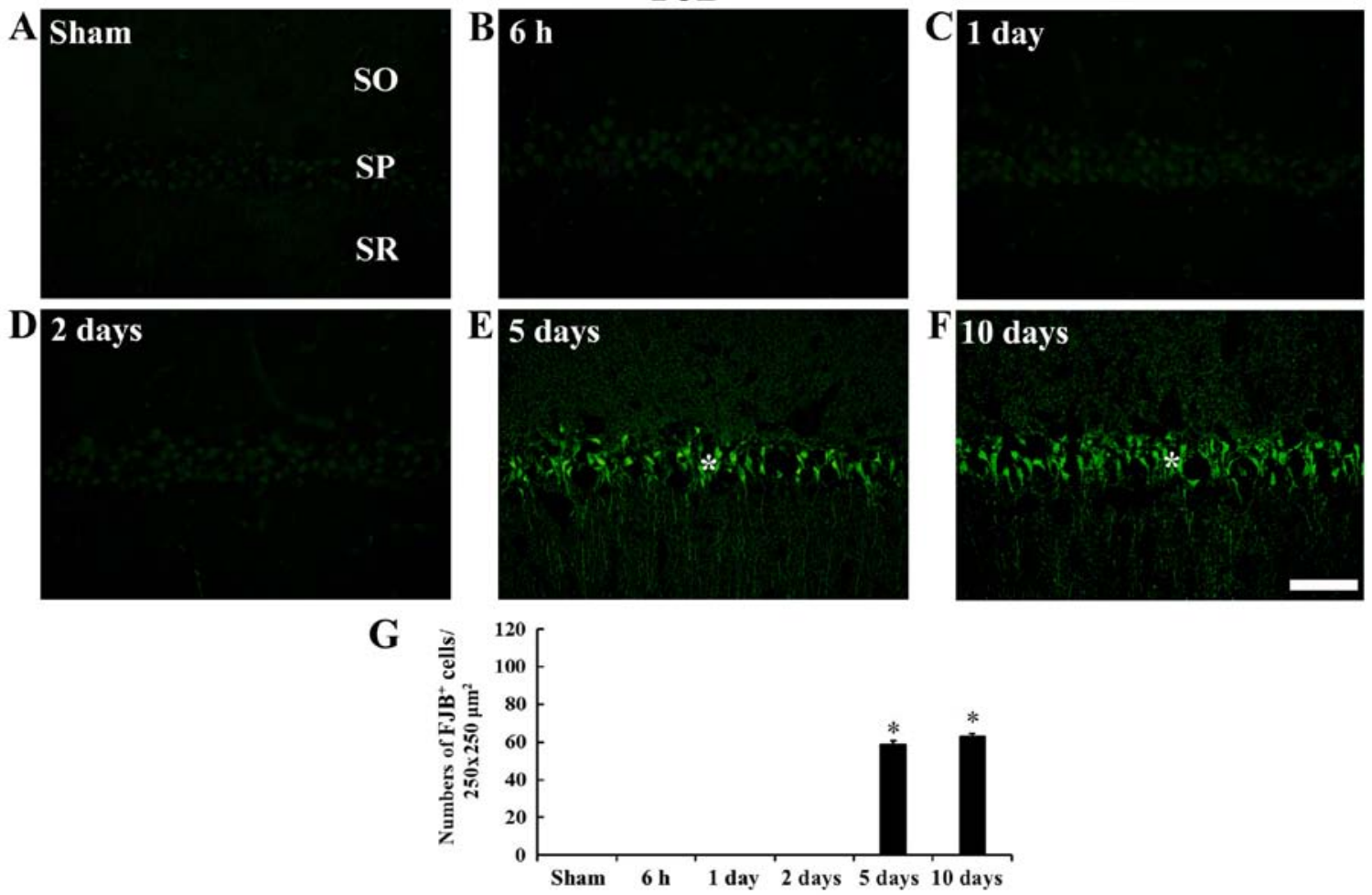

Figure 3. FJB histofluorescence staining. FJB histofluorescence staining in the CA1 field of the (A) sham group and ischemia operated groups at (B) $6 \mathrm{~h}$, (C) 1 day, (D) 2 days, (E) 5 days and (F) 10 days after tgCI. FJB positive cells are not detected in the SP until 2 days after tgCI. However, FJB positive cells (indicated by asterisks) are detected in the SP at 5 and 10 days after tgCI. Scale bar, $50 \mu \mathrm{m}$. (G) Numbers of FJB positive cells in the CA1 field (n=7 at each point in time). " $\mathrm{P}<0.05$ vs. the sham operated gerbils. The bars indicate the mean \pm standard error of the mean. SP, stratum pyramidale; FJB, Fluoro-Jade B; tgCI, transient global cerebral ischemia.

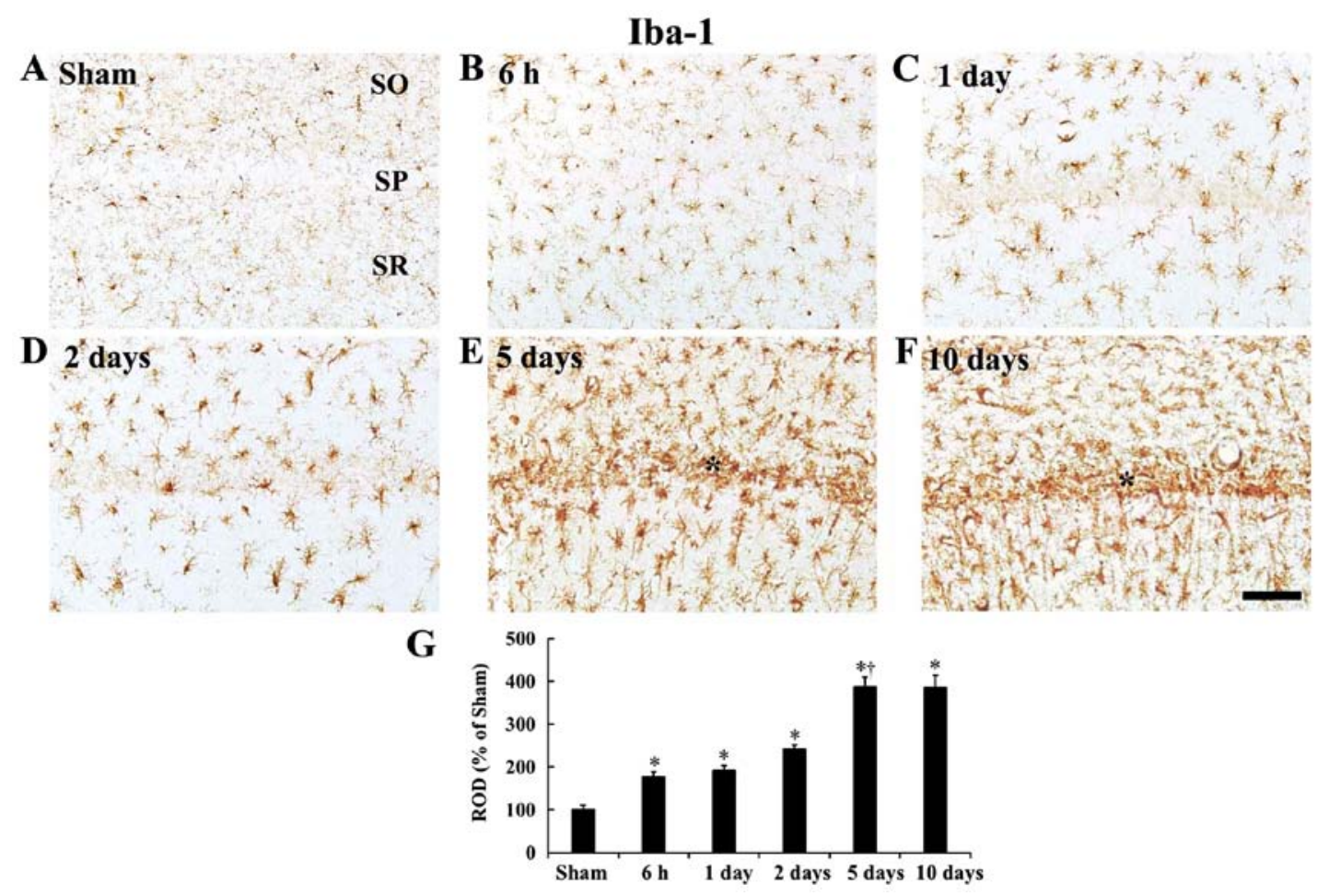

Figure 4. Iba-1 immunohistochemistry. (A-F) Ibal immunohistochemistry in the CA1 field of the (A) sham group and ischemia operated groups at (B) $6 \mathrm{~h}$, (C) 1 day, (D) 2 days, (E) 5 days and (F) 10 days after tgCI. In the ischemia operated gerbils, Iba1 immunoreactivity is gradually increased after tgCI. In particular, Iba1 immunoreactive microglia are aggregated in the SP (asterisks) at 5 and 10 days after tgCI. Scale bar, $50 \mu \mathrm{m}$. (G) ROD as \% of Iba1 immunoreactive structures after $\operatorname{tg} \mathrm{CI}\left(\mathrm{n}=7\right.$ at each point in time). ${ }^{*} \mathrm{P}<0.05$ vs. the sham operated gerbils; ${ }^{\dagger} \mathrm{P}<0.05$ vs. the pre-time-point gerbils. Bars indicate the mean \pm standard error of the mean. SP, stratum pyramidale; tgCI, transient global cerebral ischemia; ROD, relative optical density; Iba1, ionized calcium-binding adapter molecule 1 . 


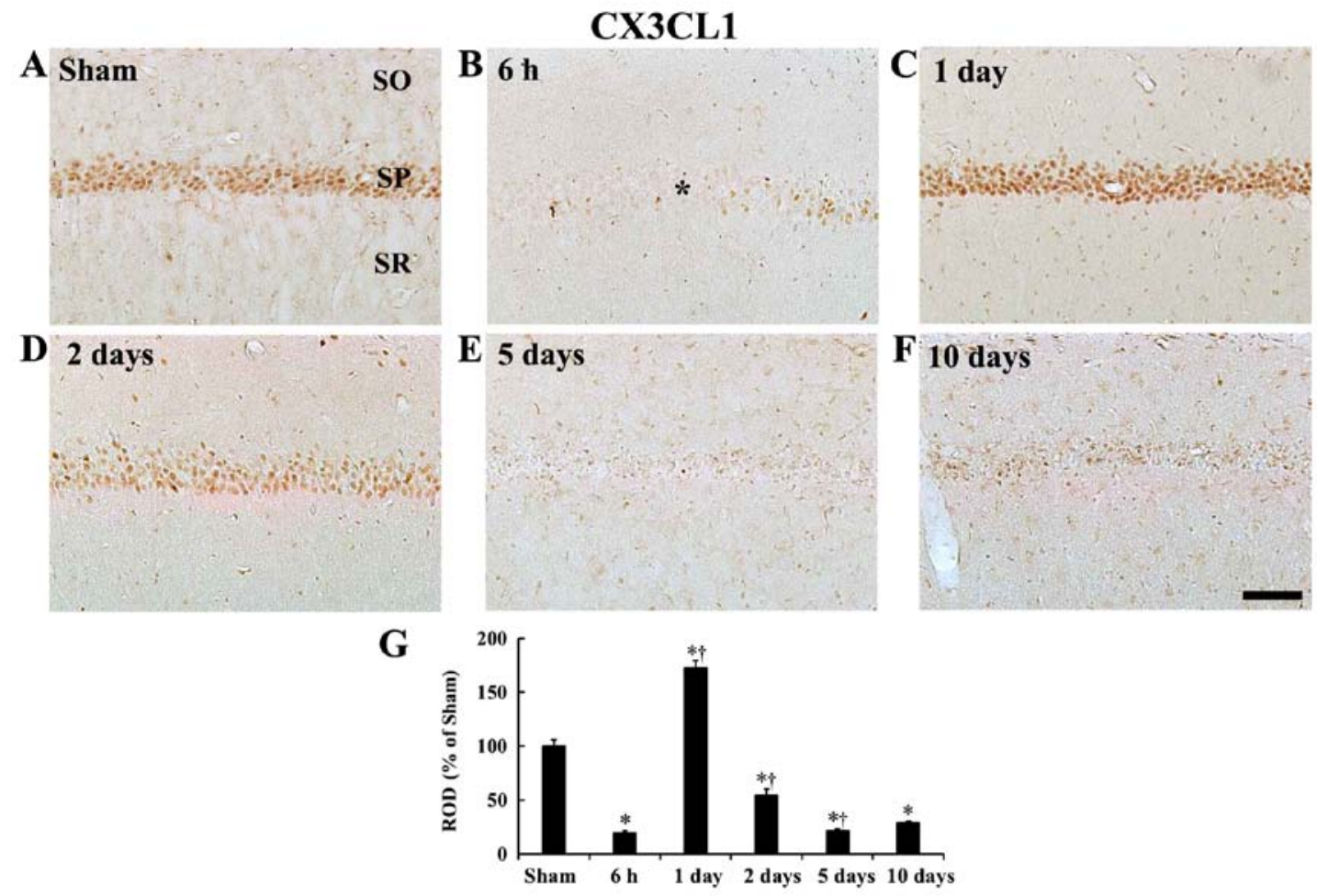

Figure 5. CX3CL1 immunohistochemistry. (A-F) CX3CL1 immunohistochemistry in the CA1 field of the (A) sham group and ischemia operated groups at (B) $6 \mathrm{~h}$, (C) 1 day, (D) 2 days, (E) 5 days and (F) 10 days after tgCI. Strong CX3CL1 immunoreactivity is shown in CA1 pyramidal cells of the SP at sham and 1 day after tgCI. CX3CL1 immunoreactivity is very weak in the CA1 field at $6 \mathrm{~h}$ (asterisk), 5 days and 10 days after tgCI. Scale bar, $50 \mu \mathrm{m}$. (G) ROD as $\%$ of CX3CL1 immunoreactive structures in the CA1 field after $\operatorname{tgCI}\left(\mathrm{n}=7\right.$ at each point in time) ${ }^{~} \mathrm{P}<0.05$ vs. the sham operated gerbils; ${ }^{\dagger} \mathrm{P}<0.05$ vs. the pre-time-point group. Bars indicate the means \pm standard error of the mean. SP, stratum pyramidale; ROD, relative optical density; tgCI, transient global cerebral ischemia; CX3CL1, C-X3-C motif ligand 1; CX3CR1, CX3C chemokine receptor 1.
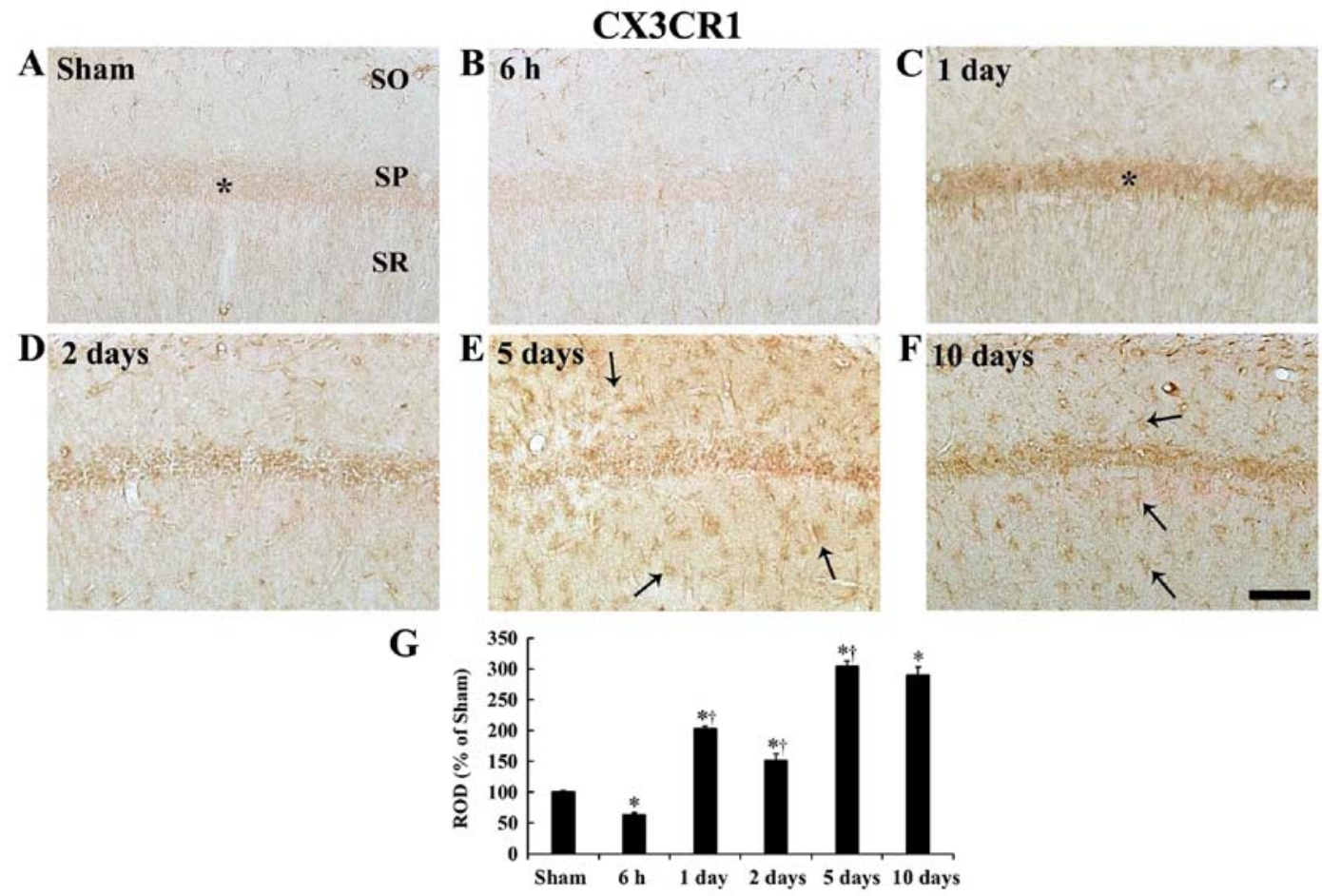

Figure 6. CX3CR1 immunohistochemistry. (A-F) CX3CR1 immunohistochemistry in the CA1 field of the (A) sham group and ischemia operated groups at (B) $6 \mathrm{~h}$, (C) 1 day, (D) 2 days, (E) 5 days and (F) 10 days after tgCI. Weak CX3CR1 immunoreactivity is detected in the SP (asterisk) of the sham operated gerbils and CX3CR1 immunoreactivity in the SP (asterisk) at 1 day after tgCI is strong. At 5 and 10 days after tgCI, strong CX3CR1 immunoreactivity is shown in cells (arrows) in the SO and SR. Scale bar, $50 \mu \mathrm{m}$. (G) ROD as \% of CX3CR1-immunoreactive structures in the CA1 field after tgCI (n=7 at each point in time). ${ }^{*} \mathrm{P}<0.05$ vs. the sham operated gerbils; ${ }^{\dagger} \mathrm{P}<0.05$ vs. the pre-time-point group. Bars indicate the mean \pm standard error of the mean. SO, stratum oriens; SP, stratum pyramidale; SR, stratum radiatum; tgCI, transient global cerebral ischemia; CX3CL1, C-X3-C motif ligand 1; CX3CR1, CX3C chemokine receptor 1 ; ROD, relative optical density. 

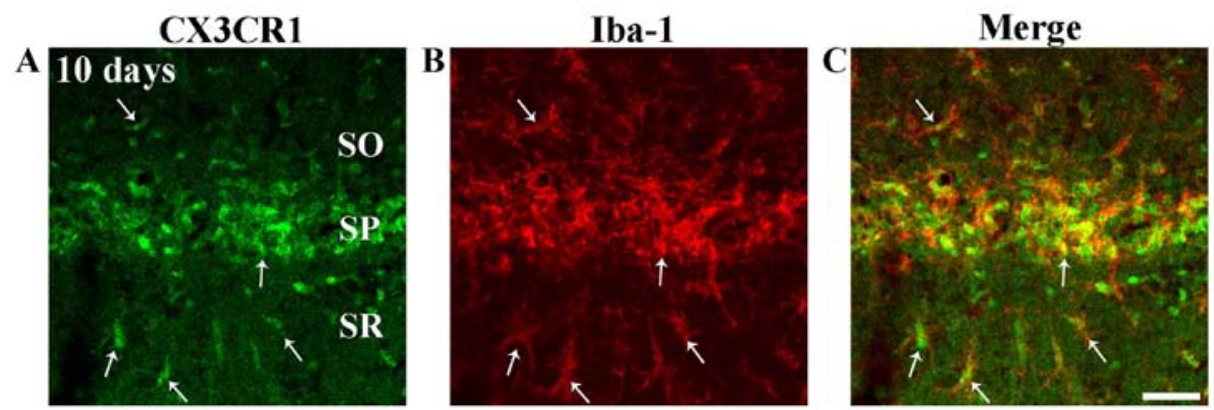

Figure 7. Double immunofluorescence staining for (A) CX3CR1 (green), (B) Iba1 (red) and (C) merged images in the CA1 field at 10 days after tgCI. CX3CR1 immunoreactivity is identified as Iba1-immunoreactive microglia (arrows). Scale bar, $50 \mu \mathrm{m}$. CX3CL1, C-X3-C motif ligand 1; CX3CR1, CX3C chemokine receptor 1; tgCI, transient global cerebral ischemia; Ibal, ionized calcium-binding adapter molecule 1.

A number of studies have reported roles of CX3CL1/CX3CR1 signaling pathway in the brain following ischemic insults. However, relevant findings have been controversial. Certain studies have demonstrated beneficial effects of CX3CL1 against cerebral ischemic damage and shown that administration of CX3CL1 can decrease ischemia-induced infarct size, neuronal death and neurologic deficits in the rat brain following focal cerebral ischemia $(34,35)$. On the other hand, it has been reported that transient focal cerebral ischemia-induced cerebral infarction is significantly lower in CX3CL1-deficient mice compared with their wild-type littermates (36). Regarding CX3CR1, its deficiency provides a neuroprotective effect against ischemia-induced brain injury in mice following focal cerebral ischemia $(18,22,37)$. However, a recent study has shown that CX3CR1 deficiency does not affect lesion size after transient focal cerebral ischemia in mice (38).

Liu et al (21) have demonstrated the detrimental effects of CX3CL1/CX3CR1-mediated microglial activation in the ischemic mouse brain. They reported that reduced expression of CX3CR1 by CX3CR1 small interfering RNA could remarkably decrease expression levels of proinflammatory cytokines, such as tumor necrosis factor- $\alpha$, interleukin (IL)- $1 \beta$ and IL- 6 , in a mouse brain after bilateral common carotid artery stenosis as well as in BV2 microglia with oxygen-glucose deprivation. In addition, certain studies have suggested detrimental roles of CX3CL1/CX3CR1 pathway in ischemic brain injury. For example, CX3CL1- and CX3CR1-knockout mice show less severe brain damage after focal cerebral ischemia $(34,39)$. In the present study, it was found that microglia began to be activated and increased in number from $6 \mathrm{~h}$ after tgCI. In addition, it was found that CX3CR1 immunoreactivity was increased in microglia at 5 and 10 days after tgCI. It is known that CX3CL1 signals to microglia by binding to CX3CR1 which functions as an inhibitor of microglial activity $(15,20)$. Therefore, a transient reduction of CX3CL1 and CX3CR1 immunoreactivities in the CA1 field at $6 \mathrm{~h}$ after tgCI might be related to the beginning of ischemia-induced microglial activation and recruitment while increases of CX3CL1 and CX3CR1 immunoreactivities at 1 day after tgCI might be a compensatory response to regulate microglial activation. Thereafter, a gradual decrease of CX3CL1 and CX3CR1 immunoreactivities in the CA1 field at 2 days after tgCI might be associated with a failure of a compensatory response, which led to considerable microglial activation. However, there might be other mechanisms to explain elevations of CX3CL1 and CX3CR1 immunoreactivities in CA1 pyramidal cells at 1 day after tgCI. Yeo et al (40) have reported that CX3CR1 immunoreactivity is transiently increased in hippocampal pyramidal cells following pilocarpine-induced status epilepticus and suggested that a transient upregulation of neuronal CX3CR1 expression might play a role in neurodegeneration following status epilepticus. In addition, a recent study has shown that CX3CR1 is upregulated in ischemic hippocampal neurons under oxygen-glucose deprivation as well as in ischemic neurons of mice subjected to focal cerebral ischemia (22). That study has suggested that neuronal CX3CR1 elevation might be associated with ischemia-induced apoptotic neuronal death (22). Based on the results of previous studies, it can be postulated that transient decreases and subsequent increases of CX3CL1 and CX3CR1 immunoreactivities in the hippocampal CA1 field at early time after tgCI might be closely associated with tgCI-induced microglial activation as well as tgCI-induced delayed neuronal death.

In this study, weak CX3CL1 immunoreactivity in CA1 pyramidal cells was detected from 5 days after tgCI. The reduction of CX3CL1 immunoreactivity in the CA1 pyramidal cells might be associated with the process of delayed neuronal death of CA1 pyramidal cells following tgCI. On the other hand, CX3CR1 immunoreactivity was increased in microglial cells from 5 days after $\operatorname{tgCI}$. It is known that cerebral ischemia leads to microglial activation including an increase of CX3CR1 expression in microglia, which causes an increase in the generation of proinflammatory cytokines in the activated microglia (21). In addition, other studies and the authors' previous study have shown marked microglial activation in the hippocampal CA1 field when tgCI-induced delayed neuronal death occurs, suggesting that activated microglia participate in phagocytic action (7,41-43). Thus, sustained and increased CX3CR1 expression in activated microglia might participate in the neuroinflammatory response and phagocytosis of activated microglia in ischemic regions.

However, there are still some important limitations of this study. As described above, a number of previous studies have reported various effects of CX3CL1-and/or CX3CR1-deficiency against ischemia-induced brain injury following focal cerebral ischemia, not tgCI, using CX3CL1 and CX3CR1 mutant mice. Therefore, a further study needs to investigate functional effects of CX3CL1 and/or CX3CR1 following tgCI using CX3CL1 and CX3CR1 mutant mice. In addition, possible upstream regulators of CX3CL1 and CX3CR1 in rodent brains following tgCI have not been fully elucidated yet. Therefore, the possible upstream regulators, which are related to the tgCI-induced changes of CX3CR1 and CX3CL1 in the hippocampus, should be also investigated in a further study. 
In conclusion, CX3CL1 and CX3CR1 immunoreactivities were markedly changed in CA1 pyramidal cells and microglia in the hippocampal CA1 field after $\operatorname{tg} \mathrm{CI}$, indicating that tgCI-induced changes in CX3CL1 and CX3CR1 expression might be closely associated with tgCI-induced delayed neuronal death and microglial activation in ischemic brain regions.

\section{Acknowledgements}

Not applicable.

\section{Funding}

This study was supported by the Bio \& Medical Technology Development Program of the NRF funded by the Korean government, MSIP (grant no. NRF-2015M3A9B6066835) and by Basic Science Research Program through the National Research Foundation of Korea funded by the Ministry of Education (grant no. NRF-2017R1D1A1B03029311).

\section{Availability of data and materials}

All data generated or analyzed during this study are included in this published article.

\section{Authors' contributions}

JHA, MHW and CHL were responsible for experimental design, data collection, data analysis and manuscript writing. JHP and TKL performed the experiments, and DWK and HAL performed data analysis and provided critical comments on the whole process of this study.

\section{Ethics approval and consent to participate}

Experimental procedures for this study were approved by the Institutional Animal Care and Use Committee at Kangwon National University (approval number: KW-180124-1).

\section{Patient consent for publication}

Not applicable.

\section{Competing interests}

The authors declare that they have no competing interests.

\section{References}

1. Arundine $\mathrm{M}$ and Tymianski M: Molecular mechanisms of glutamate-dependent neurodegeneration in ischemia and traumatic brain injury. Cell Mol Life Sci 61: 657-668, 2004.

2. Lee JC, Kim IH, Park JH, Ahn JH, Cho JH, Cho GS, Tae HJ, Chen $\mathrm{BH}$, Yan BC, Yoo KY, et al: Ischemic preconditioning protects hippocampal pyramidal neurons from transient ischemic injury via the attenuation of oxidative damage through upregulating heme oxygenase-1. Free Radic Biol Med 79: 78-90, 2015.

3. Park JH, Kim YH, Ahn JH, Choi SY, Hong S, Kim SK, Kang IJ, Kim YM, Lee TK, Won MH and Lee CH: Atomoxetine protects against NMDA receptor-mediated hippocampal neuronal death following transient global cerebral ischemia. Curr Neurovasc Res 14: 158-168, 2017
4. Rastogi L, Godbole MM, Ray M, Rathore P, Rathore P, Pradhan S, Gupta SK and Pandey CM: Reduction in oxidative stress and cell death explains hypothyroidism induced neuroprotection subsequent to ischemia/reperfusion insult. Exp Neurol 200: 290-300, 2006.

5. Barone FC and Feuerstein GZ: Inflammatory mediators and stroke: New opportunities for novel therapeutics. J Cereb Blood Flow Metab 19: 819-834, 1999.

6. Fujiwara N, Som AT, Pham LD, Lee BJ, Mandeville ET, Lo EH and Arai K: A free radical scavenger edaravone suppresses systemic inflammatory responses in a rat transient focal ischemia model. Neurosci Lett 633: 7-13, 2016.

7. Lee CH, Yoo KY, Choi JH, Park OK, Hwang IK, Kim SK, Kang IJ, Kim YM and Won MH: Neuronal damage is much delayed and microgliosis is more severe in the aged hippocampus induced by transient cerebral ischemia compared to the adult hippocampus. J Neurol Sci 294: 1-6, 2010.

8. Saito K, Suyama K, Nishida K, Sei Y and Basile AS: Early increases in TNF-alpha, IL-6 and IL-1 beta levels following transient cerebral ischemia in gerbil brain. Neurosci Lett 206: 149-152, 1996.

9. Chen Y, Hallenbeck JM, Ruetzler C, Bol D, Thomas K, Berman NE and Vogel SN: Overexpression of monocyte chemoattractant protein 1 in the brain exacerbates ischemic brain injury and is associated with recruitment of inflammatory cells. J Cereb Blood Flow Metab 23: 748-755, 2003.

10. Garau A, Bertini R, Colotta F, Casilli F, Bigini P, Cagnotto A, Mennini T, Ghezzi P and Villa P: Neuroprotection with the CXCL8 inhibitor repertaxin in transient brain ischemia. Cytokine 30: 125-131, 2005.

11. Schilling M, Strecker JK, Ringelstein EB, Schäbitz WR and Kiefer R: The role of CC chemokine receptor 2 on microglia activation and blood-borne cell recruitment after transient focal cerebral ischemia in mice. Brain Res 1289: 79-84, 2009.

12. Nishiyori A, Minami M, Ohtani Y, Takami S, Yamamoto J, Kawaguchi N, Kume T, Akaike A and Satoh M: Localization of fractalkine and CX3CR1 mRNAs in rat brain: Does fractalkine play a role in signaling from neuron to microglia? FEBS Lett 429: 167-172, 1998 .

13. Al Mamun A, Yu H, Romana S and Liu F: Inflammatory responses are sex specific in chronic hypoxic-ischemic encephalopathy. Cell Transplant 27: 1328-1339, 2018.

14. Jiang T, Zhang L, Pan X, Zheng H, Chen X, Li L, Luo J and Hu X: Physical exercise improves cognitive function together with microglia phenotype modulation and remyelination in chronic cerebral hypoperfusion. Front Cell Neurosci 11: 404, 2017.

15. Harrison JK, Jiang Y, Chen S, Xia Y, Maciejewski D, McNamara RK, Streit WJ, Salafranca MN, Adhikari S, Thompson DA, et al: Role for neuronally derived fractalkine in mediating interactions between neurons and CX3CR1-expressing microglia. Proc Natl Acad Sci USA 95: 10896-10901, 1998.

16. Chapman GA, Moores K, Harrison D, Campbell CA, Stewart BR and Strijbos PJ: Fractalkine cleavage from neuronal membranes represents an acute event in the inflammatory response to excitotoxic brain damage. J Neurosci 20: RC87, 2000.

17. Tarozzo G, Campanella M, Ghiani M, Bulfone A and Beltramo M: Expression of fractalkine and its receptor, CX3CR1, in response to ischaemia-reperfusion brain injury in the rat. Eur J Neurosci 15: 1663-1668, 2002.

18. Dénes A, Ferenczi S, Halász J, Környei Z and Kovács KJ: Role of CX3CR1 (fractalkine receptor) in brain damage and inflammation induced by focal cerebral ischemia in mouse. J Cereb Blood Flow Metab 28: 1707-1721, 2008.

19. Pabon MM, Bachstetter AD, Hudson CE, Gemma $\mathrm{C}$ and BickfordPC: CX3CL1 reduces neurotoxicity and microglial activation in a rat model of Parkinson's disease. J Neuroinflammation 8: 9, 2011.

20. Ransohoff RM, Liu L and Cardona AE: Chemokines and chemokine receptors: Multipurpose players in neuroinflammation. Int Rev Neurobiol 82: 187-204, 2007.

21. Liu Y, Wu XM, Luo QQ, Huang S, Yang QW, Wang FX, Ke Y and Qian ZM: CX3CL1/CX3CR1-mediated microglia activation plays a detrimental role in ischemic mice brain via p38MAPK/PKC pathway. J Cereb Blood Flow Metab 35: 1623-1631, 2015.

22. Wang J, Gan Y, Han P, Yin J, Liu Q, Ghanian S, Gao F, Gong G and Tang $\mathrm{Z}$ : Ischemia-induced neuronal cell death is mediated by chemokine receptor CX3CR1. Sci Rep 8: 556, 2018.

23. Zhu J, Zhou Z, Liu Y and Zheng J: Fractalkine and CX3CR1 are involved in the migration of intravenously grafted human bone marrow stromal cells toward ischemic brain lesion in rats. Brain Res 1287: 173-183, 2009. 
24. Kirino T: Delayed neuronal death in the gerbil hippocampus following ischemia. Brain Res 239: 57-69, 1982.

25. Onken M, Berger S and Kristian T: Simple model of forebrain ischemia in mouse. J Neurosci Methods 204: 254-261, 2012.

26. Park JH, Park O, Cho JH, Chen BH, Kim IH, Ahn JH, Lee JC, Yan BC, Yoo KY, Lee CH, et al: Anti-inflammatory effect of tanshinone I in neuroprotection against cerebral ischemia-reperfusion injury in the gerbil hippocampus. Neurochem Res 39: $1300-1312,2014$

27. Park JH, Shin BN, Ahn JH, Cho JH, Kim IH, Kim DW, Won MH, Hong $\mathrm{S}$, Cho $\mathrm{JH}$ and Lee $\mathrm{CH}$ : Ischemia-induced changes of PRAS40 and p-PRAS40 immunoreactivities in the gerbil hippocampal CA1 region after transient cerebral ischemia. Cell Mol Neurobiol 36: 821-828, 2016.

28. Kaufman GD, Shinder ME and Perachio AA: Correlation of fos expression and circling asymmetry during gerbil vestibular compensation. Brain Res 817: 246-255, 1999.

29. Du X, Wang D, Li Y, Huo X, Li C, Lu J, Wang Y, Guo M and Chen Z: Newly breeding an inbred strain of ischemia-prone mongolian gerbils and its reproduction and genetic characteristics. Exp Anim 67: 83-90, 2018.

30. Zhu XL, Yan BC, Tang C, Qiu GW, Wu Y, Wang J and Bo P: Neuroprotective effect of paeoniae radix rubra on hippocampal CA1 region of mice induced by transient focal cerebral ischemia via anti-gliosis and anti-oxidant activity. Chin Herb Medicines 11: 86-91, 2019.

31. Ahn JH, Shin BN, Park JH, Kim IH, Cho JH, Chen B, Lee TK, Tae HJ, Lee JC, Cho JH, et al: Long-term observation of neuronal degeneration and microgliosis in the gerbil dentate gyrus after transient cerebral ischemia. J Neurol Sci 363: 21-26, 2016.

32. Schmued LC and Hopkins KJ: Fluoro-Jade B: A high affinity fluorescent marker for the localization of neuronal degeneration. Brain Res 874: 123-130, 2000.

33. Meucci O, Fatatis A, Simen AA and Miller RJ: Expression of CX3CR1 chemokine receptors on neurons and their role in neuronal survival. Proc Natl Acad Sci USA 97: 8075-8080, 2000.

34. Cipriani R, Villa P, Chece G, Lauro C, Paladini A, Micotti E, Perego C, De Simoni MG, Fredholm BB, Eusebi F and Limatola C: CX3CL1 is neuroprotective in permanent focal cerebral ischemia in rodents. J Neurosci 31: 16327-16335, 2011.

35. Qin W, Li Z, Luo S, Wu R, Pei Z and Huang R: Exogenous fractalkine enhances proliferation of endothelial cells, promotes migration of endothelial progenitor cells and improves neurological deficits in a rat model of ischemic stroke. Neurosci Lett 569: 80-84, 2014
36. Soriano SG, Amaravadi LS, Wang YF, Zhou H, Yu GX, Tonra JR, Fairchild-Huntress V, Fang Q, Dunmore JH, Huszar D and Pan Y: Mice deficient in fractalkine are less susceptible to cerebral ischemia-reperfusion injury. J Neuroimmunol 125 : 59-65, 2002.

37. Tang Z, Gan Y, Liu Q, Yin JX, Liu Q, Shi J and Shi FD: CX3CR1 deficiency suppresses activation and neurotoxicity of microglia/macrophage in experimental ischemic stroke. J Neuroinflammation 11: 26, 2014.

38. van der Maten G, Henck V, Wieloch $T$ and Ruscher $\mathrm{K}$ : CX3C chemokine receptor 1 deficiency modulates microglia morphology but does not affect lesion size and short-term deficits after experimental stroke. BMC Neurosci 18: 11, 2017.

39. Fumagalli S, Perego C, Ortolano F and De Simoni MG: CX3CR1 deficiency induces an early protective inflammatory environment in ischemic mice. Glia 61: 827-842, 2013.

40. Yeo SI, Kim JE, Ryu HJ, Seo CH, Lee BC, Choi IG, Kim DS and Kang TC: The roles of fractalkine/CX3CR1 system in neuronal death following pilocarpine-induced status epilepticus. J Neuroimmunol 234: 93-102, 2011.

41. Nitatori T, Sato N, Waguri S, Karasawa Y, Araki H, Shibanai K, Kominami E and Uchiyama Y: Delayed neuronal death in the CA1 pyramidal cell layer of the gerbil hippocampus following transient ischemia is apoptosis. J Neurosci 15: 1001-1011, 1995.

42. Yan BC, Ohk TG, Ahn JH, Park JH, Chen BH, Lee JC, Lee CH, Shin MC, Hwang IK, Moon SM, et al: Differences in neuronal damage and gliosis in the hippocampus between young and adult gerbils induced by long duration of transient cerebral ischemia. J Neurol Sci 337: 129-136, 2014.

43. Sugawara T, Lewén A, Noshita N, Gasche Y and Chan PH: Effects of global ischemia duration on neuronal, astroglial, oligodendroglial, and microglial reactions in the vulnerable hippocampal CA1 subregion in rats. J Neurotrauma 19: 85-98, 2002.

This work is licensed under a Creative Commons Attribution-NonCommercial-NoDerivatives 4.0 International (CC BY-NC-ND 4.0) License. 